\title{
Exploration of Technical Difficulties in 3d Animation Production of Molds
}

\author{
Chen Ye \\ School of Arts, Anhui Polytechnic University, Wuhu, Anhui, 241000, China
}

Keywords: 3d animation, 3d modeling, Software carrier

\begin{abstract}
With the rapid development of science and technology, 3d animation technology is widely used in various fields, including the field of education. This paper takes the development of a major mold course of CAI courseware in a university as an example to explore the technical difficulties in the production of mold $3 \mathrm{~d}$ animation. The main research contents include the selection of software carrier, the format conversion, the $3 \mathrm{~d}$ modeling of mold parts, the production of animation, the processing of materials and lighting, and the animation rendering for reference.
\end{abstract}

\section{Introduction}

In order to implement the requirements of education reform, a mold teaching and research section of a university applied to the school for a scientific research project, which was entitled The Development of CAI Courseware in Major Mold Courses. After the project was approved, it continued to develop and has achieved some results so far. Its achievements include 3d assembly model and animation library of multiple sets of molds, and it has trained many mold design talents with high comprehensive quality. At the beginning of the project, the teaching and research section faced many challenges, among which, the technical issues had a greater impact. The teaching and research section must break through the limitations of traditional technical methods to ensure the production quality of $3 \mathrm{~d}$ animation molds. Therefore, it is very important to explore the technical difficulties in 3d animation production of molds.

\section{The Selection of Suitable Software Carrier}

The 3d animation production of mold is composed of two parts, namely the 3d modeling based on mold and the generation of animation. At the same time, the animation should be endowed with a sense of visual impact, which can bring people a good viewing experience. In the era of information technology, computer network technology has developed by leaps and bounds, and various soft wares have emerged to meet the needs of all walks of life. Therefore, before making the three-dimensional animation of the mold, it is very important to choose a suitable software carrier. Only by making a good choice of software can the subsequent work be carried out smoothly.

At present, the CAD software is widely used in the mold production field. The main reason is that these soft wares have strong $3 \mathrm{~d}$ modeling function. But there are problems with weak animation simulation capabilities and poor animation production functions, so it is difficult to achieve the expected purpose as a software carrier. On the contrary, 3dMAX, MAYA and other software are quite different from CAD software. While they have the function of animation production, their 3d modeling ability is not prominent enough, and their effect is limited when used alone ${ }^{[1]}$.

It can be seen from the above analysis that CAD software and 3dMAX software have their own advantages and disadvantages, so the integrated use of the two as a software carrier can make its role fully exerted. After some discussion, the teaching and research section decided to use the UG in the CAD software to create a three-dimensional model, and then use the 3dMAX software to animate it. Through the addition of transparency, lighting and materials, the three-dimensional animation related to the mold was finally obtained.

UG is a type of CAD software developed by UGS in the United States. Since its appearance, this software has been used in the field of automobile and civil airliner manufacturing, and has achieved good application results. The software is mature and has many modeling functions, such as 3d entity, 
features and free-form surface modeling functions. As a software carrier for modeling, it can reduce the difficulty of modeling, so it is recognized and preferred by the relevant industry. The 3dMAX is a software owned by Autodesk, which integrates various functions, including modeling, animation rendering and animation production. Because of this, it has become the most popular software.

Taking the above two kinds of software as the main choice is conducive to the effective development of the project, and at the same time, it can train the practical ability of the participants. After the completion of the project, the abilities of the participants will be improved, which is conducive to the promotion of professional mold education and teaching.

\section{The Format Conversion}

According to the above analysis, considering the advantages and disadvantages of each software, two kinds of software are selected as the carrier, UG and 3dMAX respectively. UG is firstly applied to generate 3d solid model, and then 3dMAX software is used to animate and render it, so as to give full play to the role of both. However, how to convert the file format has become another technical difficulty. After practical research, the teaching and research section learned that the STL format is a universal format and can be supported by the selected software. Therefore, the STL format was chosen as the main choice to overcome the technical difficulties of format conversion. The steps are as follows:

(1) Use the UG software to create the model, if it is a built model, use the software to open it;

(2) After opening the software, click the options successively, and the system will automatically pop up the dialog box shown in Figure 1. After the selection is completed, click OK with the mouse;

(3) In the dialog box, specify the name of the output file, which aims to change the output format of the model file into STL to achieve the expected purpose;

(4) Input the corresponding title according to the system prompt;

(5) After the selector pops up, click the $3 \mathrm{~d}$ shape of the mold with the mouse, and then click OK, then the suffix of the model file will become XXX.STL;

(6) Use the mouse to click on the 3dMAX software;

(7) Click the Import option in the 3dMAX software;

(8) After clicking the above options, a dialog box will pop up, and then click SteroeLitho to convert the format of the $3 \mathrm{~d}$ mold model. After the conversion, the file format will be changed to STL;

(9) In the process of format conversion, the parameters must be set, but only the Smooth Angle needs to be modified. The main reason is that this parameter can control the smoothness of the graph. If the parameter is unreasonable, it will affect the quality of the model. Generally, the value of this parameter will exceed 50;

(10) Click OK with the left mouse button, and the 3dMAX software will successfully receive the three-dimensional model of the mold;

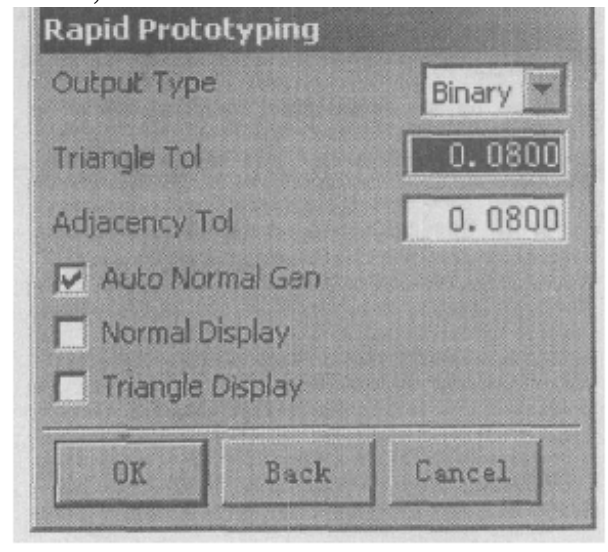

Fig.1 Rapid-Protoyping Dialog Box 
After the above processing, the mold graphics will inevitably lose some information. For example, open the model with UG software, you can see the complete three-dimensional model, which when you use 3dMAX software to open the file, the model will become a curved surface one. However, this change is difficult to be perceived by naked eyes, so the impact on project implementation is negligible ${ }^{[2]}$.

\section{The 3d Modeling of Mold Parts}

It is known that the mold parts have the characteristics of complexity, such as core cavity, sheet metal parts, and so on. Its production and molding involved in many methods, including solid modeling and sheet metal molding, so that the organic integration of these methods can reduce the difficulty of $3 \mathrm{~d}$ mold construction. However, in the process of modeling, only knowing the molding method can not meet the requirements of modeling. It is also necessary to master the software operation methods, especially the using of UG software. If the modeler has little knowledge about it, it is difficult to make full use of it and the software can not play the real role in this process. After in-depth research, the teaching and research group made the participants understand and master the application methods of the software through education and training before the project started ${ }^{[3]}$.

There are many parts of the mold, and the process of modeling is the assembly of many parts. Therefore, the animation obtained finally is the assembly process of parts. In this case, the formation of the mold assembly is a technical problem. At present, there are two commonly used design methods, namely bottom-up and top-down. The former refers to designing parts before assembling and then assembling in a reasonable order. The latter refers to the method of clarifying the relationship between components and assembly, and then designing components according to the model.

\section{The Production of Animation}

The linear movement of mold parts is a common part of mold animation. It is relatively simple to use 3dMAX software to create such animation. However, 3dMAX software will be unable to handle those complex animation, such as stretching process, plastic mold plastic flow and sheet bending, etc. Next, the sheet bending animation is used as an example to analyze the methods and steps to solve the problem.

(1) Complete the construction of the $3 \mathrm{~d}$ model of the curved plate. If the model is relatively simple, you don't need to use UG software, you can directly build the model in the 3dMAX software.

(2) Use the Modify tool in the 3dMAX software to modify the length, width and height of the plate piece. It is worth noting that the number of modified sections should not exceed 100 . After processing, the sheet metal parts will be decomposed into multiple volumes and change from the whole to a variable object, thus creating favorable conditions for the subsequent work.

(3) If the created bending part model has multiple angles, it is particularly critical to determine the bending order. It is known that the outer corner is bent first and the inner corner is bent later. If the order and the principle are inconsistent, the bending action cannot be achieved.

(4) With the help of the bending command in the Modify tool, the design of the bending parameters of the model can be completed, which mainly include bending degree, bending direction, bending radius, etc.;

(5) In Track Viewd, the action key frames can be adjusted to try to match the bending in the animation with the actual bending.

For the mold model created by the UG software, the number of segments cannot be modified in the 3dMAX software. In short, the bending action cannot be achieved. Therefore, the teaching and research section adopted 3dMAX software to establish a cuboid. This cuboid has a number of segments compared to the ordinary model. Using this as a carrier of the mold model, the bending action of the mold can be realized.

In the production of animation, the participants also applied numerous techniques to make the 
animation more visual. In addition, in the process of the mold opening and closing movements, various forms of movement will appear, such as side core pulling, fixed distance parting, etc. If there is only one movement, it is less difficult to achieve. However, the simultaneous existence of multiple actions has become a technical problem that plagues the participants. Eventually, the participants completed the group and key frame settings according to the organization, so that this problem was overcome ${ }^{[4]}$.

\section{The Processing of Materials and Lighting}

The scientific and reasonable choice of cutting method is an effective means to enhance the visual effect. In addition, the processing of materials and lights is also very critical.

In the actual process, it is found that if there are only one part to be processed, there is no difficulty. However, through the above analysis, it can be seen that the mold is a combination of many parts and many parts need to be processed, so the difficulty in the actual processing stage is relatively big and it cannot meet the actual requirements. To solve the problem, the teaching and research section has adopted the following measures:

(1) There is no need to deal with screws and pins. The main reason is that screws and pins play a smaller role in the mold. They have the same function, and are less related to the principle to be expressed by the animation. Therefore, it is OK not to deal with the parts and lights when they are processed.

(2) Formulate attachment rules for materials and colors. For example, metal parts can be given copper gold, while plastic parts can be given eye-catching red and so on. The implementation of this rule greatly reduces the difficulty of processing, but under special circumstances, the application effect of this processing method will be affected, and participants need to make adjustments flexibly.

(3) For some molds that are more complex in structure and difficult to process effectively, the teaching and research section invites professionals to process the materials and lights of the mold from an aesthetic point of view to enhance the expressive power of animation.

\section{Animation Rendering}

After the above processing is completed, animation rendering can be performed, and the rendering effect is closely related to the quality and size of the animation. As a professional animation software, 3dMAX has many output formats to choose from. Among the many animation formats, the AVI format is used frequently. This format has strong applicability. Most media playback software in the world supports the AVI format and Windows operation. The player that comes with the system is no exception, so the teaching and research section has chosen the AVI format as the main choice.

It is known that the number of mold animations produced by the teaching and research section is up to 129. If the traditional compression technology is used, the size of each animation is $12 \mathrm{M}$. After calculation, the space occupied by 129 animations is as high as 1548M. The storage capacity of ordinary optical discs is about $700 \mathrm{M}$, which does not meet the actual requirements, so the teaching and research section uses 360 compression software to compress the animation. At the same time, remove the background image in the animation, and choose a resolution of $640 \times 480$ to compress the size of the animation.

After processing, the compressed size of each animation is changed from $12 \mathrm{M}$ to $1 \mathrm{M}$, and one fourth of the disc capacity is enough to save all the animations.

\section{Conclusion}

To sum up, under the background of the new era, China's economy has achieved rapid development and the processing and manufacturing industry has made an indelible contribution. Molds are critical to the manufacturing industry, and are related to the quality of manufacturing. In 
this context, it is suggested that relevant colleges and enterprises attach importance to the role of computer software, so as to give full play to its advantages, and to promote the development of related industries. Mold 3d animation production is an effective way of computer software application. In the actual application process, animation producers should take measures to overcome the technical problems in the selection of software carrier, the format conversion, the 3d modeling of mold parts, the production of animation, the processing of materials and lighting, and the animation rendering, so as to ensure the quality and application of animation.

\section{Acknowledgment}

Key Humanities and Social Sciences Project of Anhui Education Department: Research on Strategies for Upgrading Cultural Creativity of Urban Outdoor Advertising (Project No. SK2013A023).

\section{References}

[1] Shang Aipeng, Li Pengfei, Zhong Fei. Application of Cluster Rendering in Vehicle 3d Animation [J]. Electric Locomotive and City Rail Vehicle, 2020,43 (02) : 75-77.

[2] Zhou Xiaoying, Lv Wenwen. Practice of Virtual Reality Technology in 3d Animation [J]. Cradle of Journalists, 2020 (03) : 80-81.

[3] Duan Zhongyuan, Liu Xinyu. Application of 3d Game Engine to Animation Production Technology in the Context of Virtual Reality [J]. Science and Technology Communication, 2020,12 (02) : 144-145.

[4] Pan He, Shen Junfeng. Technical Difficulties and Flow Control of Application-oriented Animation [J]. Science and Technology Innovation \& Application, 2017 (16) : 106. 